\title{
MARKOVIAN ANALYSIS OF COVID-19 DYNAMICS
}

\author{
S. Ibrahim-Tiamiyu ${ }^{1}$, O. V. Oni ${ }^{2}$ and E. O. Adeleke ${ }^{3}$ \\ ${ }^{1}$ Department of Statistics, Federal College of Animal Health and Production Technology, \\ Ibadan, Nigeria \\ ${ }^{2}$ Department of Statistics, Federal College of Animal Health and Production Technology, \\ Ibadan, Nigeria \\ ${ }^{3}$ Department of Mathematics, Federal University of Agriculture, Abeokuta
}

\begin{abstract}
Cite this article: S. IbrahimTiamiyu, O. V. Oni, E. O. Adeleke (2021), Markovian Analysis of Covid-19 Dynamics. African Journal of Mathematics and Statistics Studies 4(1), 94-106. DOI: 10.52589/ajmss_fzzq3wga.
\end{abstract}

DOI: 10.52589/

ajmss_fzzq3wga

Available Online at: https://doi.org/10.52589/ajmss _fzzq3wga

Received: 11 Jan 2021

Accepted: 4 Feb 2021

Published: 18 April 2021

Copyright $\odot 2020$ The Author(s). This is an Open Access article distributed under the terms of Creative Commons Attribution-

NonCommercial-

NoDerivatives 4.0 International (CC BY-NC-ND 4.0), which permits anyone to share, use, reproduce and redistribute in any medium, provided the original author and source are credited.
ABSTRACT: Covid-19 is an emergency and viral infection with its outbreak being termed as one of the great epidemics in the 21st century causing so many deaths, which made WHO declare it as a pandemic emergency. This virus is new and comes with its characteristics of which randomness and uncertainty are among its common features. In this paper, we developed a model for carrying out an analysis of COVID-19 dynamics using Markovchain theory methodology. Here, we employed the use of conditional probability distribution as embedded in the Markov property of our chain to construct the transition probabilities that were used in determining the probability distributions of COVID19 patients as well as predicting its future spread dynamics. We provide a step-by-step approach to obtaining probability distributions of infected and recovered individuals, of infected and recovering and of a recovered patient being getting infected again. This study reveals that irrespective of the initial state of health of an individual, we will always have probabilities $P_{R I} /\left(P_{I R}+P_{R I}\right)$ of an individual being infected and $P_{R I} /\left(P_{I R}+P_{R I}\right)$ of an individual recovering from this disease. Also, with increasing ' $n$ ', we have an equilibrium that does not depend on the initial conditions, the implication of which is that at some point in time, the situation stabilizes and the distribution $X_{n+1}$ is the same as that of $X_{n}$. We envision that the output of this model will assist those in the health system and related fields to plan for the potential impact of the pandemic and its peak.

KEYWORDS: COVID-19, Markov-chain, Markov Property, Transitional Probability Matrix, Probability Distribution 


\section{INTRODUCTION}

COVID-19 is an emergent viral infectious disease which was first reported in December 2019 in a city in the Chinese province of Hubei, Wuhan; the viral aetiology of this infection known as COVID-19 virus belongs to the Betacoronavirus genus which is known for causing respiratory (if not gastrointestinal or neurological) diseases in both humans and animals $[1,2]$.

Its outbreak has been termed as one of the great epidemics in the 21 st century causing over 700,000 deaths with well over 21 million of reported cases of infection throughout 188 countries/regions of the world [2,3]. In March 2020, Nigeria reported its first case, since then there have been over 53,000 confirmed cases of covid-19, including 1,011 deaths with $1.9 \%$ CFR [4].

This is a new virus and a completely new situation; so it exhibits its particular characteristics and there may be the need for a model to be developed to tackle the real situation; to evaluate the spread of the disease within a discrete-time interval, to provide answers to such questions as "How many individuals will be infected per day, week, month, etc.?", will the present situation reach equilibrium (in the long-run)? How many people will remain infected or recovered? The knowledge of which will help the health system and the authorities involved to plan for the potential impact of the pandemic. Also, it is essential to estimate the number of infections to be able to analyse the spread of the disease.

In the last few decades, there were several major outbreaks of infectious diseases such as SARS (2003), HIN influenza in 2009, H7N9 influenza in 2013, Ebola in Democratic Republic of Congo in 1995. Various mathematical models have been used to determine the trend of disease spread and provide optimal control strategies. [5] and [6] developed ordinary differential equations stochastic SEIR models to study the dynamics of infectious disease and control interventions using the outbreak of Ebola in DRC as a case study, while for Lassa fever [7] modified SEIR model using a system of ordinary differential equations to analyse the stabilities of the Dynamical System with the addition of demographic effects.

Meanwhile, the theory of Markov Chain to epidemiology is not new [8,9] since the outbreak of COVID-19, a lot of work has been done: [10] developed a discrete-time stochastic epidemic model with binomial distributions to study the transmission of the disease. The estimated model parameters based on fitting to newly reported data and simulation show the newly confirmed cases will continue to decline and the total confirmed cases will reach the peak in February with application to COVID-19 cases in China.

Also [11] compared the distribution of COVID-19 cases based on daily reported data in Iran using Normal, Log-normal, and Weibull, their results showed that Weibull distribution was the best fit with the data. But, parameters of distributions were different between new cases and the daily deaths data.

In this paper, we develop a model based on Markov chain theory methodology that can be used to determine the probability distributions of COVID-19 patients as well as to predict its future spread dynamics. Every forecasting model has its advantages and speciality for solving complex real-world problems but Markov chains, which is a special case of the stochastic process [12] with the Markov property, excels, in that it offers ideal conditions for the study of mathematical modelling of various phenomena depending on random-variables [13]. The Markov property makes it possible to simplify some predictions about stochastic processes by 
viewing the future as being independent of the past given the present state of the process $[14,15]$. This property makes the Markov chain adaptable to a situation like this when epidemic data is still insufficient and difficult to apply directly to existing mathematical models which requires relatively large sample data.

Also, the tractability and relative ease of calculating quantities of interest make Markov modelling attractive in the study of disease dynamics which are often characterized by disease states. Here, we made use of discrete, first-order Markov to provide a step-by-step approach to obtaining probability distributions of infected and recovered individuals, when an infected will recover and a recovered individual may likely get infected again. The knowledge of this is useful for characterizing the disease and its course, especially in assisting the policymakers and the general population to be more prepared as well as promoting more logical actions.

\section{METHODOLOGY}

The Markov process, which incorporates the stochastic element, is being employed as the main component of this model to determine the various probability distributions of infected and recovered individuals and the possible transitions for an individual from one state of health to another. Here, the probability distribution over the state space at a certain response occasion depends only on the recent health condition of an individual. That is, to determine the probability distribution of an individual's next state of health, we only need information about the recent state of health. This is referred to as the memoryless or Markovian property i.e.,

$$
\begin{array}{r}
P\left\{X_{n+1}=i_{n+1} \mid X_{n}=i_{n}, X_{n-1}=i_{n-1}, \cdots\right\} \\
=P\left\{X_{n+1}=i_{n+1} \mid X_{n}=i_{n}\right\}
\end{array}
$$

With this, the states of health of an individual before ' $n$ ' are assumed to be irrelevant to the state of health at occasion ' $n+1$ ', that is, only the most recent outcome is of use in predicting the next outcome.

The Markov chain becomes homogeneous, if

$$
\begin{gathered}
P\left\{X_{n+1}=i_{n+1} \mid X_{n}=i_{n}, X_{n-1}=i_{n-1}, \cdots, X_{0}=i_{0}\right\} \\
=P\left\{X_{n+1}=i_{n+1} \mid X_{n}=i_{n}\right\}
\end{gathered}
$$

is independent of ' $n$ '.

Majorly, we are interested in using this principle of Markov chain to study the behaviour of system $X_{n, n \geq 0, n<\infty}$ as it settles down into a regular pattern.

In this model and relation to the known characteristics of the COVID-19 pandemic, we assume that an individual is in one of the following states: Infected or Recovered Infectious (denoted by I). This is the first state where it is assumed that an individual has finished the incubation period, may infect other people and start developing clinical signs. It is also assumed that only an individual with medium symptoms can get to this state, not those that will die. After this state, an individual can remain or move on to Recovered State. 
Recovered State: This is the state where it is assumed that an infectious individual survived the disease but may likely be infected again or develop a natural immunity to the virus.

From this, we have the following transition probabilities

$$
\begin{aligned}
& P\left\{X_{n+1}=R \mid X_{n}=I\right\}=P_{I R} \\
& P\left\{X_{n+1}=I \mid X_{n}=R\right\}=P_{R I}
\end{aligned}
$$

Similarly,

$$
P\left\{X_{n+1}=I \mid X_{n}=I\right\}=1-P_{I R}
$$

and

$$
P\left\{X_{n+1}=R \mid X_{n}=R\right\}=1-P_{R I}
$$

\begin{tabular}{cc|cc} 
& & $I$ & $R$ \\
\cline { 2 - 4 }$\underset{\mathrm{n}}{\text { Transitio }}$ & $I$ & $1-P_{I R}$ & $P_{I R}$ \\
$\underset{\substack{\text { probabili } \\
\text { ty }}}{\text { matrix: }}$ & $R$ & $P_{R I}$ & $-P_{R I}$
\end{tabular}

and its graph becomes

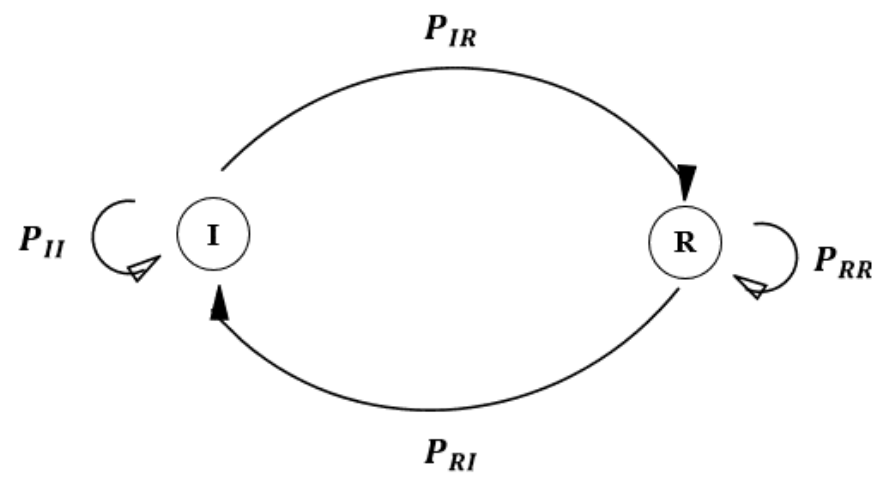

with another assumption that the probability of being in the state $I$ initially is $\lambda_{0}(I)$ and that of being in state $R$ is $\lambda_{0}(R)$.

That is,

$$
P\left(X_{0}=I\right)=\lambda_{0}(I)
$$

and 


$$
P\left(X_{0}=R\right)=\lambda_{0}(R)
$$

by law of Total Probability,

$$
P\left(X_{0}=I\right)+P\left(X_{0}=R\right)=1
$$

this implies that $\lambda_{0}(I)+\lambda_{0}(R)=1$

$$
\lambda_{0}(R)=1-\lambda_{0}(I)
$$

We employ the use of the following theorems to establish our results.

Theorem 1.1 [15]. A Markov chain $\left\{X_{n}, n \geq 0\right\}$ is completely characterised by the initial distribution $V$ and the transition probability matrix $P$.

$$
\begin{array}{ll}
\text { Proof. } & P\left\{X_{0}=i_{0}\right\}=V_{i_{0}} \text { by definition of } V, P\left\{X_{0}=i_{0}, X_{1}=\right. \\
\left.i_{1}\right\}=P\left\{X_{1}=i_{1} \mid X_{0}=i_{0} \cdot P X_{0}=i_{0}\right\}=P_{i_{0} i_{1}} \cdot V_{i_{0}} \text { by definition of } P=V_{i_{0}} \cdot P_{i_{0} i_{1}}
\end{array}
$$

Now, by the induction hypothesis, assume that

$$
P\left\{X_{0}=i_{0}, \cdots, X_{k}=i_{k}\right\}=V_{i_{0}} P_{i_{0} i_{1}}, P_{i_{0} i_{1}}, P_{i_{1} i_{2}}, \cdots, P_{i_{k-1} i_{k}} \text { for } k=1,2, \cdots, n-1 .
$$

We shall show that it is true for $k=n$.

$$
\begin{gathered}
P\left\{X_{0}=i_{0}, \cdots, X_{n}=i_{n}=P\left\{X_{n}=i_{n} \mid X_{n-1}=i_{n-1}, \cdots, X_{0}\right.\right. \\
\left.=i_{0}\right\} \cdot P\left\{X_{0}=i_{0}, \cdots, X_{n-1}=i_{n-1}\right\} P\left\{X_{n}=i_{n} \mid X_{n-1}=i_{n-1}\right\} \cdot P\left\{X_{0}\right. \\
\left.=i_{0}, \ldots, X_{n-1}=i_{n-1}\right\} \text { by Markov property. } \\
=P_{i_{n-1}, i_{n}} \cdot V_{i_{0}} P_{i_{0}}, i, \cdots, P_{i_{n-1}, i_{n-1}} \text { by time homogeneity } \\
=V_{i_{0}} P_{i_{0}, i} \cdots P_{i_{n-1}, i_{n}}
\end{gathered}
$$

This theorem is proved by induction.

Theorem 1.2 (Chapman Kolmogorov Equation ( [15]).

$$
P_{i j}^{(n)}=\sum_{r \in S} P_{i r}^{(k)} \cdot P_{r j}^{(n-k)} \text { where } k \text { is a fixed integer such that } 0 \leq k \leq n .
$$

Proof. Fix an integer ' $k$ ' such that $0 \leq k \leq n$. Then, 


$$
\begin{aligned}
P_{i j}^{(n)}=P\left\{X_{n}=\right. & \left.j \mid X_{0}=i\right\}=\sum P\left\{X_{0}=j, X_{k}=r \mid X_{0}=i\right\} \\
& =\sum_{r \in S} P\left\{X_{n}=j \mid X_{k}=r, X_{0}=i\right\} \cdot P\left\{X_{k}=r \quad X_{0}=i\right\} \\
& =\sum_{r \in S} P\left\{X_{n}=j \mid X_{k}=r\right\} \cdot P\left\{X_{k}=r \quad X_{0}=i\right\} \text { from Markov's property } \\
& =\sum_{r \in S} P\left\{X_{n-k}=j \mid X_{0}=r\right\} \cdot P\left\{X_{k}=r \quad X_{0}=i\right\} \text { from time } \\
& - \text { homogeneity }=\sum_{r \in S} P_{i j}^{(n-k)} \cdot P_{i r}^{(k)} \text { by the fact that } P_{i j}^{(n)} \\
& =P\left\{X_{n}=j \mid X_{0}=i\right\} \forall i, j \in S
\end{aligned}
$$

Introducing the matrix notation, we have:

$$
P^{(n)}=\left[P_{i j}^{(n)}\right]
$$

Theorem 1.3. ([15]).

$$
V_{n}=V P^{n}
$$

Proof. Using

$$
\begin{aligned}
& \quad V_{i}^{(n)}=P\left\{X_{n}=j \mid X_{0}=i\right\} \cdot P\left\{X_{0}=i\right\} \\
& =\sum_{i \in S} P\left\{X_{n}=j \mid X_{0}=i\right\} V_{-} i
\end{aligned}
$$

where $\left(V_{i}\right)$ is the initial distribution. Thus, $\left(V_{i}\right)$ can be re-written in matrix form as:

$$
V^{(n)}=V^{(0)} \cdot P^{(n)}=V \cdot P^{n}
$$

This theorem establishes the fact that the $n^{\text {th }}$ power of the one-step transition probability can be used to compute the marginal distribution of $X_{n}$.

Now, to our results.

Result 1: Let $I$ and $R$ be two possible states of health of an individual, the probabilities of either being infected or recovered at a particular occasion ' $n$ ' are: 


$$
\begin{aligned}
& P\left(X_{n}=I\right)=\frac{P_{R I}}{P_{I R}+P_{R I}}+\frac{\left(1-P_{I R}-P_{R I}\right)^{n} \cdot\left[\lambda_{0}(I)\left(P_{I R}+P_{R I}\right)-P_{R I}\right]}{P_{I R}+P_{R I}} \\
& P\left(X_{n}=R\right)=\frac{P_{I R}}{P_{I R}+P_{R I}}+\frac{\left(1-P_{R I}-P_{I R}\right)^{n} \cdot\left[\lambda_{0}(I)\left(P_{I R}+P_{R I}\right)-P_{I R}\right]}{P_{I R}+P_{R I}}
\end{aligned}
$$

Proof. We have:

$$
\begin{aligned}
P\left(X_{n+1}=I\right) & =P\left\{X_{n}=I \text { and } X_{n+1}=R\right\}+P\left\{X_{n}=R \text { and } X_{(n+1)}=I\right. \\
& =P\left\{X_{n}=I\right\} \cdot P\left\{X_{n+1}=I \mid X_{n}=I\right\}+P\left\{X_{n}=R\right\} \cdot P\left\{X_{n+1}=I \mid X_{n}=R\right. \\
& =P\left\{X_{n}=I\right\} \cdot\left(1-P_{I R}\right)+P\left\{X_{n}=R\right\} \cdot P_{R I} \\
& =\left(1-P_{I R} \cdot P\left\{X_{n}=I\right\}+P\left\{X_{n}=R\right\} \cdot P_{R I}\right.
\end{aligned}
$$

By the law of total probability,

$$
\begin{aligned}
P\left\{X_{n}=R\right\}= & 1-P\left\{X_{n}=I\right\} \therefore \quad P\left\{X_{n+1}=I\right\} \\
& =\left(1-P_{I R}\right) \cdot P\left\{X_{n}=I\right\}+P_{R I}\left(1-P\left\{X_{n}-I\right\}\right) \\
& =\left(1-P_{I R}\right) \cdot P\left\{X_{n}=I\right\}-P\left\{X_{n}=I\right\} \cdot P_{R I} \\
& =P\left\{X_{n}=I\right\}\left[1-P_{I R}-P_{R I}\right]+P_{R I}
\end{aligned}
$$

$\therefore$ At occasion $n+1$, the probability of an individual becoming infected is:

$$
P\left\{X_{n+1}=I\right\}=\left(1-P_{I R}-P_{R I}\right) \cdot P\left\{X_{n}=I\right\}+P_{R I}
$$

From this, we have:

$$
\begin{aligned}
& P\left\{X_{n+1}=I\right\}=\left(1-P_{I R}-P_{R I}\right) \cdot P\left\{X_{0}=I\right\}+P_{R I} P\left\{X_{n+1}=I\right\} \\
& =\left(1-P_{I R}-P_{R I}\right) \cdot \lambda_{0}+P_{R I} P\left\{X_{2}=I\right\}=\left(1-P_{I R}-P_{R I}\right) \cdot P\left\{X_{0}=I\right\}+P_{R I} \\
& P\left\{X_{2}=I\right\}=\left(1-P_{I R}-P_{R I}\right) \cdot\left[\left(1-P_{I R}-P_{R I}\right) \cdot \lambda_{0}(I)+P_{R I}\right]+P_{R I} P\left\{X_{2}=I\right\} \\
& =\left(1-P_{I R}-P_{R I}\right)\left(1-P_{I R}-P_{R I}\right) \cdot \lambda_{0}(I)+P_{R I}\left(1-P_{I R}-P_{R I}\right)+P_{R I} \\
& =\left(1-P_{I R}-P_{R I}\right)^{2} \cdot \lambda_{0}(I)+P_{R I}\left(1-P_{I R}-P_{R I}\right)+P_{R I} \\
& P\left\{X_{3}=I\right\}=\left(1-P_{I R}-P_{R I}\right) \cdot P\left\{X_{2}=I\right\}+P_{R I} \\
& =\left(1-P_{I R}-P_{R I}\right) \cdot\left[\left(1-P_{I R}-P_{R I}\right)^{2} \cdot \lambda_{0}(I)+P_{R I}\left(1-P_{I R}-P_{R I}\right)\right]+P_{R I} \\
& =\left(1-P_{I R}-P_{R I}\right)^{3} \cdot \lambda_{0}(I)+P_{R I}\left(1-P_{I R}-P_{R I}\right)^{2}+P_{R I}\left(1-P_{I R}-P_{R I}\right)+P_{R I}
\end{aligned}
$$

By inspection, then

$$
\begin{aligned}
P\left(X_{m}=I\right)= & \left(1-P_{I R}-P_{R I}\right)^{m} \cdot \lambda_{0}(I)+P_{R I}\left(1-P_{I R}-P_{R I}\right)^{m-1}+\cdots \\
& +P_{R I}\left(1-P_{I R}-P_{R I}\right) P_{R I} \\
& =\left(1-P_{I R}-P_{R I}\right)^{m} \cdot \lambda_{0}(I)+P_{R I} \sum_{j=0}^{m-1}\left(1-P_{I R}-P_{R I}\right)^{j}
\end{aligned}
$$

using the formula for the sum of finite geometric progression, we have: 


$$
\begin{aligned}
\sum_{j=0}^{m-1}\left(1-P_{I R}-P_{R I}\right)^{j}=\frac{1-\left(1-P_{I R}-P_{R I}\right)^{m}}{P_{I R}+P_{R I}} P\left(X_{m}=I\right) \\
=\frac{P_{R I}}{P_{I R}+P_{R I}}+\left(1-P_{R I}-P_{I R}\right)^{m} \cdot \frac{\left[\lambda_{0}(I)\left(P_{I R}+P_{R I}\right)-P_{R I}\right]}{P_{I R}+P_{R I}}
\end{aligned}
$$

and by induction

$$
P\left(X_{n}=I\right)=\frac{P_{R I}}{P_{I R}+P_{R I}}+\frac{\left(1-P_{R I}-P_{I R}\right)^{n} \cdot\left[\lambda_{0}(I)\left(P_{I R}+P_{R I}\right)-P_{R I}\right]}{P_{I R}+P_{R I}}
$$

so that at any given occasion ' $n$ ' the probability of an individual becoming infectious is:

$$
P\left(X_{n}=I\right)=\frac{P_{R I}}{P_{I R}+P_{R I}}+\frac{\left(1-P_{R I}-P_{I R}\right)^{n} \cdot\left[\lambda_{0}(I)\left(P_{I R}+P_{R I}\right)-P_{R I}\right]}{P_{I R}+P_{R I}}
$$

where ' $n$ ' represents discrete time interval which may be days, weeks, or months depending on the occurrence.

Similarly, the probability of recovery at a particular $(n+1)$ occasion can be obtained as:

$$
\begin{aligned}
P\left(X_{n+1}=R\right) & =P\left(X_{n}=R \text { and } X_{n+1}=R\right)+P\left(X_{n} \text { and } X_{n+1}=R\right) P\left(X_{n+1}=R\right) \\
& =P\left(X_{n}=R\right) \cdot P\left(X_{n+1}=R \mid X_{n}=R\right)+P\left(X_{n}=I\right) \cdot P\left(X_{n+1}=R \mid X_{n}=I\right) \\
& \therefore \quad P\left(X_{n+1}=R\right)=P\left(X_{n}=R\right) \cdot\left(1-P_{R I}\right)+P\left(X_{n}=I\right) \cdot P_{I R}
\end{aligned}
$$

Using the law of total probability

$$
\begin{aligned}
P\left(X_{n}=I\right)=1 & -P\left(X_{n}=R\right) P\left(X_{n+1}=R\right)=P\left(X_{n}=R\right)\left(1-P_{R I}\right)+\left(1-P\left(X_{n}=R\right)\right) \cdot P_{I R} \\
& =\left(1-P_{R I}\right) P\left(X_{n}=R\right)+P_{I R}-P\left(X_{n}=R\right) \cdot P_{I R} P\left(X_{n+1}=R\right) \\
& =\left(1-P_{R I}-P_{I R}\right)
\end{aligned}
$$

where; $P\left(X_{0}=R\right)=\lambda_{0}(R)$

From this, 


$$
\begin{aligned}
& P\left(X_{m}=R\right)=\left(1-P_{R I}-P_{I R}\right)^{m} \cdot \lambda_{0}(R)+P_{I R}\left(1-P_{R I}-P_{I R}\right)^{m-1}+\cdots+P_{I R}\left(1-P_{R I}-P_{I R}\right) \\
& =\left(1-P_{R I}-P_{I R}\right)^{m} \cdot \lambda_{0}(R)+\sum_{j=0}^{m-1}\left(1-P_{R I}-P_{I R}\right) \cdot P_{I R} \\
& =\left(1-P_{R I}-P_{I R}\right)^{m} \cdot \lambda_{0}(R)+P_{I R} \sum_{j=0}^{m-1}\left(1-P_{R I}-P_{I R}\right)^{j} \\
& \sum_{j=0}^{m-1}\left(1-P_{R I}-P_{I R}\right)^{j}=\frac{1-\left(1-P_{R I}-P_{I R}\right)^{m}}{P_{I R}+P_{R I}} \\
& \therefore \quad P\left(X_{m}=R\right)=\left(1-P_{R I}-P_{I R}\right)^{m} \cdot \lambda_{0}(R)+\frac{P_{I R}\left(1-\left(1-P_{R I}-P_{I R}\right)^{m}\right)}{P_{I R}+P_{R I}} \\
& =\frac{\left(P_{I R}+P_{R I}\right) \cdot\left(1-P_{R I}-P_{I R}\right)^{m} \cdot \lambda_{0}(R)+P_{I R}-P I R\left(1-P_{R I}-P_{I R}\right)^{m}}{P_{I R}+P_{R I}} \\
& =\frac{P_{I R}}{P_{I R}+P_{R I}}+\frac{\left(1-P_{R I}-P_{I R}\right)^{m}\left[\lambda_{0}(R)\left(P_{I R}+P I R\right)-P_{R I}\right]}{P_{I R}+P_{R I}} \\
& P\left(X_{m}=R\right)=\frac{P_{I R}}{P_{I R}+P_{R I}}+\frac{\left(1-P_{R I}-P_{I R}\right)^{m}\left[\lambda_{0}(R)\left(P_{I R}+P_{R I}\right)-P_{I R}\right]}{P_{I R}+P_{R I}} \\
& P\left\{X_{1}=R\right\} \quad=\left(1-P_{R I}-P_{I R}\right) \cdot P\left\{X_{0}=R\right\}+P_{I R} \\
& =(1-P R I-P I R) \cdot \lambda 0+P I R \\
& P\left\{X_{2}=R\right\} \quad=\left(1-P_{R I}-P_{I R}\right) \cdot P\left\{X_{1}=R\right\}+P_{I R} \\
& =\left(1-P_{R I}-P_{I R}\right)\left[\left(1-P_{R I}-P_{I R}\right) \cdot P\left(X_{0}=R\right)+P_{I R}\right]+P_{I R} \\
& =(1-P R I-P I R)(1-P R I-P I R) \cdot \lambda 0(R)+P I R(1-P R I-P I R)+P I R \\
& =(1-P R I-P I R) 2 \cdot \lambda 0(R)+P I R(1-P R I-P I R)+P I R \\
& P\left\{X_{3}=R\right\} \quad=\left(1-P_{R I}-P_{I R}\right) \cdot P\left\{X_{2}=R\right\}+P_{I R} \\
& =(1-P R I-P I R)[(1-P R I-P I R) 2 \cdot P(X 0=R)+P I R(1-P R I-P I R) \\
& +P I R]+P I R \\
& =(1-P R I-P I R) 3 \cdot P(X 0=R)+P I R(1-P R I-P I R) 2+P I R(1-P R I \\
& -P I R)+P I R \\
& =(1-P R I-P I R) 3 \cdot \lambda 0(R)+P I R(1-P R I-P I R) 2+P I R(1-P R I- \\
& P I R)+P I R
\end{aligned}
$$


By inspection,

By induction

$$
P\left(X_{n}=R\right)=\frac{P_{I R}}{P_{I R}+P_{R I}}+\frac{\left(1-P_{R I}-P_{I R}\right)^{n}\left[\lambda_{0}(R)\left(P_{I R}+P_{R I}\right)-P_{I R}\right]}{P_{I R}+P_{R I}}
$$

$\therefore$ The probability that an individual will recover from a particular occasion ' $n$ ' is:

$$
P\left(X_{n}=R\right)=\frac{P_{I R}}{P_{I R}+P_{R I}}+\frac{\left(1-P_{R I}-P_{I R}\right)^{n}\left[\lambda_{0}(R)\left(P_{I R}+P_{R I}\right)-P_{I R}\right]}{P_{I R}+P_{R I}}
$$

At times, it may be of interest to obtain the probability distribution or to estimate the number of infected individuals that may likely recover after ' $n$ ' steps (' $n$ ' number of times) or those that might have recovered but may likely be infected again, to this we have the following result:

The result I: In ' $n$ ' number of times, the probabilities of an infected individual being recovered and that of the recovered individual being infected are:

$$
P^{n}(I, R)=\frac{P_{I R}}{P_{I R}+P_{R I}}-\frac{\left(1-P_{R I}-P_{I R}\right)^{n} \cdot P_{I R}}{P_{I R}+P_{R I}}
$$

and

$$
P^{n}(R, I)=\frac{P_{R I}}{P_{I R}+P_{R I}}-\frac{\left(1-P_{I R}-P_{R I}\right)^{n} \cdot P_{R I}}{P_{I R}+P_{R I}}
$$

Proof. In equation,

$$
P\left\{X_{n}=R\right\}=\frac{P_{I R}}{P_{I R}+P_{R I}}+\frac{\left(1-P_{R I}-P_{I R}\right)^{n}\left[\lambda_{0}(R)\left(P_{I R}+P_{R I}\right)-P_{I R}\right]}{P_{I R}+P_{R I}}
$$

Set $\lambda_{0}(R)=0$

$$
\begin{aligned}
P^{n}[I, R] & =\frac{P_{I R}}{P_{I R}+P_{R I}}+\frac{\left(1-P_{R I}-P_{I R}\right)^{n}\left[0 \cdot\left(P_{I R}+P_{R I}\right)-P_{I R}\right]}{P_{I R}+P_{R I}} \\
& =\frac{P_{I R}}{P_{I R}+P_{R I}}-\frac{\left(1-P_{R I}-P_{I R}\right)^{n} \cdot P_{I R}}{P_{I R}+P_{R I}}
\end{aligned}
$$

while the probability that an individual who has recovered can get infected again can thus be obtained by setting $\lambda_{0}(I)=0$ in the equation

$$
\begin{aligned}
P\left\{X_{n}=I\right\} & =\frac{P_{R I}}{P_{I R}+P_{R I}}+\frac{\left(1-P_{I R}-P_{R I}\right)^{n}\left[\lambda_{0}(I)\left(P_{I R}+P_{R I}\right)-P_{R I}\right]}{P_{I R}+P_{R I}} \\
\therefore \quad P^{n}(R, I) & =\frac{P_{R I}}{P_{I R}+P_{R I}}+\frac{\left(1-P_{I R}-P_{R I}\right)^{n}\left[0 \cdot\left(P_{I R}+P_{R I}\right)-P_{R I}\right]}{P_{I R}+P_{R I}} \\
& =\frac{P_{R I}}{P_{I R}+P_{R I}}+\frac{\left(1-P_{I R}-P_{R I}\right)^{n} \cdot\left(-P_{R I}\right)}{P_{I R}+P_{R I}} \\
& =\frac{P_{R I}}{P_{I R}+P_{R I}}-\frac{P_{R I}\left(1-P_{I R}-P_{R I}\right)^{n}}{P_{I R}+P_{R I}}
\end{aligned}
$$


Result II: In ' $n$ ' number of times, the probabilities of an infected person remaining infectious and that of a recovered person remaining in recovery state are:

$$
\begin{aligned}
P^{n}(I, I) & =\frac{P_{R I}}{P_{I R}+P_{R I}}+\frac{P_{I R}\left(1-P_{I R}-P_{R I}\right)^{n}}{P_{I R}+P_{R I}} \\
P^{n}(R, R) & =\frac{P_{I R}}{P_{I R}+P_{R I}}-\frac{P_{R I}\left(1-P_{R I}-P_{I R}\right)^{n}}{P_{I R}+P_{R I}}
\end{aligned}
$$

Proof. In equation,

$$
P\left(X_{n}=I\right)=\frac{P_{R I}}{P_{I R}+P_{R I}}+\frac{\left(1-P_{I R}-P_{R I}\right)^{n}\left[\lambda_{0}(I) \cdot\left(P_{I R}+P_{R I}\right)-P_{I R}\right]}{P_{I R}+P_{R I}}
$$

Set $\lambda_{0}(I)=1$

$\therefore P(I, I) \quad=\quad$ Probability of an individual remaining infected in several steps

$$
\begin{aligned}
& =\frac{P_{I R}}{P_{I R}+P_{R I}}+\frac{\left(1-P_{I R}-P_{R I}\right)^{n}\left[1 \cdot\left(P_{I R}+P_{R I}\right)-P_{R I}\right]}{P_{I R}+P_{R I}} \\
& =\frac{P_{R I}}{P_{I R}+P_{R I}}+\frac{P_{I R} \cdot\left[1-P_{I R}-P_{R I}\right]^{n}}{P_{I R}+P_{R I}}
\end{aligned}
$$

while that of a recovered individual remaining in recovered state $P^{n}(R, R)$, can be obtained by setting $\lambda_{0}(R)=L$ in equation

$$
\begin{aligned}
P\left(X_{n}=R\right) & =\frac{P_{I R}}{P_{I R}+P_{R I}}+\frac{\left(1-P_{R I}-P_{I R}\right)^{n}\left[\lambda_{0}(R)\left(P_{I R}+P_{R I}\right)-P_{I R}\right]}{P_{I R}+P_{R I}} \\
\left.\therefore \quad P^{(} R, R\right) & =\frac{P_{I R}}{P_{I R}+P_{R I}}+\frac{\left(1-P_{R I}-P_{I R}\right)^{n}\left[1 \cdot\left[P_{I R}+P_{R I}\right]-P_{I R}\right.}{P_{I R}+P_{R I}} \\
& =\frac{P_{I R}}{P_{I R}+P_{R I}}+\frac{P_{R I}\left[1-P_{R I}-P_{I R}\right]^{n}}{P_{I R}+P_{R I}}
\end{aligned}
$$

So, for large ' $n$ ', our model becomes:

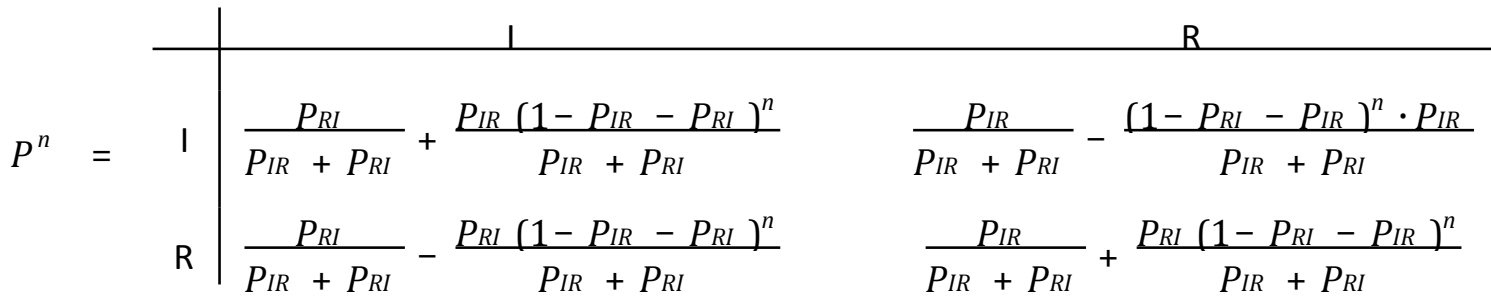

$$
\begin{aligned}
& \therefore \quad P^{n}=\frac{1}{P_{I R}+P_{R I}}\left\{\begin{array}{ll}
P_{R I} & P_{I R} \\
P_{R I} & P_{I R}
\end{array}\right\}+\left(1-P_{I R}-P_{R I}\right)^{n}\left\{\begin{array}{cc}
P_{I R} & -P_{I R} \\
-P_{R I} & P_{R I}
\end{array}\right\}
\end{aligned}
$$

In the long-run, that is, as $n \rightarrow \rightarrow \infty$, our model converges to:

$$
\lim _{n \longrightarrow \infty} P^{n}=\frac{1}{P_{I R}+P_{R I}}\left\{\begin{array}{ll}
P_{R I} & P_{I R} \\
P_{R I} & P_{I R}
\end{array}\right\}
$$


This means that each row of our model (transition matrix $P^{n}$ ) converges to an

equilibrium distribution $\left(\frac{P_{R I}}{P_{I R}+P_{R I}}, \frac{P_{I R}}{P_{I R}+P_{R I}}\right)$ as $n \longrightarrow \infty$

This shows that our $P^{n}$ converges to a fixed matrix

$$
\left(\frac{P_{R I}}{P_{I R}+P_{R I}}, \quad \frac{P_{I R}}{P_{I R}+P_{R I}}\right) \text { as } n \rightarrow \infty
$$

This implies that for large ' $n$ ' no matter which state we start in, we will always have probabilities about $\frac{P_{R I}}{P_{I R}+P_{R I}}$ of an individual being infected and $\frac{P_{I R}}{P_{I R}+P_{R I}}$ of an individual recovering from this disease.

Another implication is, we will always have an equilibrium that does not depend upon the initial conditions as the memory of the past dies out with increasing ' $n$ ', this means that at some point in time the situation stabilises and the distribution of $X_{n+1}$ will be the same as the distribution of $X_{n}$ at equilibrium.

\section{REFERENCES}

[1]. Cui J. Li and Shi Z. Li (2019 March). Origin and evolution of pathogenic coronaviruses, Nature Reviews/ Microbiology, 17, 181-193 doi:10.1038/s 41579 - 018 0118-9.

[2]. Catrin Sohrabi, Zaid Alsafi and Riaz Agha (May 2020). World Health Organization declares global emergency: A review of the 2019 novel coronavirus (COVID-19). International Journal of Surgery. London, England.

[3]. World Health Organization: Coronavirus Disease (Covid-190) Situation Report-209.

[4]. Nigeria Centre for Disease Control: COVID-19 Situation Report 182 (28 ${ }^{\text {th }}$ August 2020).

[5]. Chowell G., Castillo- Chavez C., Fenimore P., Christopher M., Kribs-Zaleta C., Arriola L. (2004). Model parameters and outbreak control for SARS, Emerg. Infect. Disease (10), 12581263.

[6]. Lekone P., Finkenstadt B., (2006). Statistical inference in a Stochastic Epidemic SEIR model with control intervention: Ebola as a case study. Biometrics.

[7]. Oni O.V, Akanle Y.O., Ibrahim-Tiamiyu S. (2018). Inclusion of Birth and Death Rate in the Modelling of Lassa fever in Nigeria. International Journal of Scientific Research in Mathematical and Statistical Sciences Vol.5, Issue-4, pp.22-32.

[8]. Alfonso Vivanco-Lira and Leon Guanajuato (2020): Predicting COVID-19 distribution in Mexico through a discrete and time-dependent Markov chain and a SIR - like the model.

[9]. Elise F. Zipkin, Christopher S. Jennelle and Evan G. Cooch (2010): A primer on the application of Markov chains to the study of wildlife disease dynamics. British Ecological S257-259. MIT Press, Boston, Massachusetts, USA. 
[10]. Sha He and Sanyi Tang (2020). A discrete Stochastic Model of the COVID-19 Outbreak: Forecast and Control. Mathematical Biosciences and Engineering, 2020 17(4): 2792 - 2804.

[11]. Gholami E., Mansori K., Soltani-Kermanshashi M. (2020). Statistical Distribution of novel coronavirus in Iran, Int. Journ. One Health, 6(2): 143 - 146.

[12]. Vidyadhar G.K. (1995). Modelling and Analysis of Stochastic Systems. Chapman and Hall/CRC17.

[13]. Kemeny J.G. and Snell J.L., (1976). Finite Markov chains. Springer - Verlag. New York; USA.

[14]. Bishop Y.M., Fienberg S. \& Holland P.W. (1971) Discrete multivariate analysis: theory in practice. Models for Measuring Change: Contingency Tables and Markov Models, pp. 257 - 259. MIT Press, Boston, Massachusetts, USA.

[15]. Elgharbi S., Esghir M., Ibrinchi O., Abarda A., El Hajji S., Elbernoussi S., Grey Markov model for the prediction of the electricity production and consumption. Springer New York; New York, NY, USA 2020; Volume 81, pp. 206-219. 